\title{
Chance, Variation and the Nature of Causality in Ecological Communities
}

\author{
Hans de Kroon and Eelke Jongejans
}

\author{
It's a coincidence, it is not scientific. \\ Major Walsh in Close Encounters of the Third Kind \\ (Steven Spielberg, director; Columbia Pictures, 1977)
}

\begin{abstract}
Chance is pervasive in nature. Erratic events such as storms and fires can cause major damage to an ecosystem. Rare successful long distance dispersal events like a viable seed landing in just the right habitat can form the stepping stone for range expansion of a plant species. Illustrated with two examples we argue that in ecology chance events are scale-dependent. We show how random stochastic variation in species interactions may result in relative stability at a higher community level. In other systems the reverse may take place, in which deterministic interactions result in unpredictable chaotic dynamics. Analysing the processes and dynamics at these different scales has led to an increasing mechanistic understanding of the variation in ecological communities in space and time. Unambiguous identification of cause and effect relations from this work is of the greatest importance, as many ecosystems in the world are not amenable to experimentation. This work should form the scientific basis for identifying the threats to ecosystems and defining proper conservation and mitigation measures.
\end{abstract}

\section{Introduction: The Fascinating Complexity of Ecosystems}

One central problem in ecology is understanding the distribution of species and individuals over the landscape. Species are organised in ecological communities of producers (generally plants) and consumers (herbivores and predators) that change across the landscape. Climatic factors and soil and water conditions may change already over short distances and vary with altitude vs latitude. Adaptations determine the distribution of species over gradients. Beautiful nature documentaries

H. de Kroon $(\bowtie) \cdot$ E. Jongejans

Faculty of Science, Departments of Plant Ecology and Animal Ecology,

Institute for Water and Wetland Research, Radboud University, Nijmegen, The Netherlands

e-mail: h.dekroon@science.ru.nl

(C) The Author(s) 2016

K. Landsman and E. van Wolde (eds.), The Challenge of Chance,

The Frontiers Collection, DOI 10.1007/978-3-319-26300-7_11 
often focus on these amazing characteristics of species by which they are able to cope with the challenges of their often extreme environment.

An important goal in ecology as a scientific discipline is understanding the driving forces, or underlying mechanisms responsible for differences in distribution of species in their natural habitats. However, how much mechanistic understanding is possible in ecosystems in which chance processes play a prominent role? For example, long-distance migration of plants is subject to the coincidental combination of a rare event like a heavy storm taking place at exactly the right time and place carrying ripe seeds to another location with exactly the right conditions for establishment. Such events are hardly tractable in the field. How much does chance affect distributions of individuals and interactions between them, and how much do actual ecological and evolutionary processes contribute? The question is important not only for the progress of ecology as a scientific discipline, but also for understanding the impact of disturbances (such as global climate change) and formulating appropriate interventions to mitigate such disturbances.

Illustrated with two examples, we argue that coincidence, variation and causality are scale-dependent. With scale we imply the extent of time and space (McGill 2010), but also the hierarchical structure of ecosystems, in which individuals of the same species are grouped within populations, populations of different species are grouped within structured ecological communities, which in turn interact with abiotic conditions regarding climate, soil and water within the landscape. Patterns expressed at one scale are driven by causal processes at a smaller underlying scale. Vice versa, random processes at a lower scale sum up to measurable variation at a higher scale. As a result, rare events at a lower scale can be predicted at a higher scale, e.g. under which climatic conditions new soybean rusts from South America can be expected in North America (Isard et al. 2011).

In the first example we give an overview on current theory explaining the maintenance of species diversity, with emphasis on hyper-diverse communities such as tropical forests. The complexity is daunting. Such communities exist of hundreds, sometimes thousands of species, each with their own characteristics, ecological relationships with other species and responses to environmental conditions. What are the stabilizing forces preventing species from extinction? How important are stabilizing forces preserving these communities relative to chance effects?

In the second example we investigate trends of populations of species over time, as they are influenced by deterministic and stochastic factors. Studying such trends is of great importance for the conservation of species and the prediction of the impact of environmental stress factors. We will see that in the currently fragmented landscapes all over the world, populations are ruled by chance events affecting the extinction of small populations, as well as rare long-distant dispersal events. How can we gain control over this stochasticity, in order to understand and predict how environmental factors influence the viability of populations? Answering this question very much depends on the spatial scale at which we are studying processes, from a very local patch of suitable habitat where a limited number of individuals survive and reproduce, to a region (such as an entire country) harbouring numerous of these small populations that together form a predictive trend. 


\section{Example 1: Explaining the Maintenance of Species Diversity}

\subsection{Coexistence Theory: Species Differ in Niches}

One of the most long-lasting questions in ecology is to explain how so many organisms can coexist in a community. Hyper-diverse communities (Box 1) are tantalizing examples challenging a long-standing paradigm in ecology. The 'competitive exclusion principle', formulated by the Russian biologist Georgy Gauss in the 1930s and based on laboratory experiments with Paramecium (unicellular ciliated protozoa), states that two species can only stably coexist if they differentiate in their fundamental requirements such as their food source (their 'niches'). Early on, the competitive exclusion principle received theoretical support from population models (Lotka 1920; Volterra 1928). The Lotka-Volterra equations describing the competition between two species and defining the conditions for competitive exclusion or stable coexistence can be considered the $\mathrm{E}=\mathrm{mc}^{2}$ of community ecology. They still form the cornerstone of modern coexistence theory (Chesson 2000).

\section{BOX 1: the dazzling number of species that coexist in natural plant communities}

Plant communities can harbour very high numbers of species in a given area. Communities differ in composition and complexity. Why are some communities more species-rich than others? Why are the tropical forests overwhelmingly species-rich and why are these levels of biodiversity not reached in the temperate or boreal forests?

The differences are enormous. Current estimates suggest the minimum number of tropical tree species in the world between 40,000 and 53,000 (Slik et al. 2015). The number of tree species described globally for temperate forest is only 1166 (Latham and Ricklefs 1993). Also at smaller scale, tropical forests can contain an astonishing number of species. For example, a single hectare (approximately one baseball field or two soccer fields) can support hundreds of species of trees (record: 942 species of trees per hectare in Amazonian Ecuador; Wilson et al. 2012). An area of the size of a fraction of the Radboud University campus would thus harbour approximately the same the number of species as the entire temperate forest region in the world including Europe, Asia and North-America $\left(4.2\right.$ million $\left.\mathrm{km}^{2}\right)$. How did this diversity arise, and how is the diversity maintained?

Extensively managed, relatively nutrient-poor grasslands all over the world are another example of extreme plant species richness, albeit at a smaller scale (Wilson et al. 2012). Per $\mathrm{m}^{2}$ such communities can have dozens of species of higher plants (record: 89 species of vascular plants $\mathrm{m}^{-2}$ for a mountain grassland in Argentina). How is it possible that such communities are maintained, without a few superior species starting to dominate and drive competitively inferior species to extinction? 
In its essence, coexistence theory states that different species in a community can stably coexist if a species gains a competitive advantage over the resident community when that species becomes rare. Consequently, if for whatever reason a species gets low in numbers, its population will bounce back resulting in coexistence. Such frequency-dependent population dynamics is only possible if species differ in their requirements to complete their life cycle, i.e. differ in their niches. Niche differences can arise from many different characteristics, with food source as the most obvious one. Differences in reproduction (the 'regeneration niche', i.e. requirements for nesting in birds, micro-climatic conditions for seedlings to establish) and natural enemies (herbivores and diseases) also constitute niche axes. What is crucial is that these differences in requirements result in differences in survival and reproductive schemes between species. Consequently, if a species becomes rare in the community, its species-specific niche 'opens up', resulting in positive population growth rates and recovery. For all species combined, niche differences are a necessary stabilising force.

\subsection{Natural Enemies as Niche-Axes: The Janzen-Connell Hypothesis}

This theory sparked a quest for important niche axes, particularly for plants for which niche differences are hard to conceive because plants all have essentially the same nutritional requirements. In the early nineteen-seventies, Daniel Janzen and Joseph Connell invoked natural enemies in explaining the high tropical tree diversity (Condit 1995; Connell 1971; Janzen 1970). Co-evolution between the feeding adaptations of herbivores and the defence mechanisms of plants has led to sophisticated adaptations resulting in numerous specific plant-herbivore relationships. In what is now known as the Janzen-Connell hypothesis, they argued that each plant species accumulates its own specific community of natural enemies, which is more detrimental to this particular plant species than to other species in the community. Consequently, offspring of a tree has relatively lower chances for establishment close to the parent tree than at further distance where other tree species are growing. A given species therefore cannot stand its local ground forever, but, Janzen and Connell hypothesized, if this is a reciprocal process applying to all species in the forest it will lead to stable coexistence of large numbers of tree species. Nearly fifty years after its conception, the Janzen-Connell hypothesis has only gained in importance in community ecology (Comita et al. 2014). Attention has shifted from aboveground herbivores to belowground enemies (root feeding larvae, worms and insects, and particularly soil pathogenic micro-organisms including bacteria, fungi and other unicellular organisms) (Mangan et al. 2010). Janzen-Connell effects are also considered an important driving force in species-rich grasslands (Bever et al. 2012; de Kroon et al. 2012; Petermann et al. 2008). 
But how can the Janzen-Connell hypothesis result in stable coexistence of many species? The reason is that Janzen-Connell effects balance competition between trees of the same species relative to competition between trees of different species. If a tree species becomes dominant it will be at disadvantage relative to other species in the community. Conversely, when a species gets rare in the community, its seedlings may easily find suitable areas for growth and the species will gain in competitive ability and abundance. Such frequency-dependent responses are consistent with the general theory of species coexistence (Chesson 2000).

\subsection{Coexistence Through Intransitive Competition and Rock-Paper-Scissor Games}

The frequency-dependent population dynamics expected from Janzen-Connell effects have been compared to intransitive competitive networks. Intransitive competition implies that competitive abilities of different species cannot be ranked along a hierarchy in which a single species gains competitive dominance (Buss 1980; Gilpin 1975). An example of an intransitive competitive network is when species $\mathrm{A}$ is superior to species $\mathrm{B}$, and $\mathrm{B}$ is superior to $\mathrm{C}$, but $\mathrm{C}$ is superior to species $\mathrm{A}(\mathrm{A}>\mathrm{B} ; \mathrm{B}>\mathrm{C} ; \mathrm{C}>\mathrm{A})$. Models of spatial distributions of individuals and populations suggest that intransitive competitive relationships result in coexisting populations (Laird and Schamp 2006). They contrast with transitive or hierarchical competition, as is predicted from competition for essential resources. Transitive competition implies that when species $\mathrm{A}$ is competitively superior to species $\mathrm{B}$, and $\mathrm{B}$ to species $\mathrm{C}$, species $\mathrm{A}$ will also win in competition with species $\mathrm{C}$ ( $\mathrm{A}>\mathrm{B}$; $\mathrm{B}>\mathrm{C}$; $=>\mathrm{A}>\mathrm{C}$ ). Indeed, if species compete for a limited soil resource, it is inconceivable that a superior competitor $\mathrm{A}$ that wins in competition with a species $\mathrm{B}$ will lose in competition with species $\mathrm{C}$ that is itself competitively inferior to species B (de Kroon et al. 2012; Lankau 2010). However, species-specific belowground interactions may result in species interactions consistent with intransitive competitive networks (Lankau et al. 2011).

Intransitive competition is also referred to as a rock-paper-scissor game. This concept has been developed as an example of game theory (Nowak and Sigmund 2004; Weitzel and Rosenkranz, this volume), and it is easily conceivable because you play it with your kids. Rock wins from scissor, scissor wins from paper, paper wins from rock, there is not a single winner. If each player makes one of the three choices completely at random, independently from what the other players chose or have chosen, and is therefore unable to predict any of the other players, all players will end with a similar proportion of runs won. The trick is to predict a pattern of choice with your competitors, which is never completely random but is for instance based on previous choices. 


\subsection{Tests with Bacterial Communities: Rock-Paper-Scissor Dynamics Is not Enough for Stable Coexistence}

How can a rock-paper-scissor game based on unpredictable interactions among the players result in stable (i.e. predictable) coexistence of the players themselves? It should be realized that in ecology the play is implemented somewhat differently from human politics and economics. In a sizable ecological community the number of players (i.e. individuals) is almost infinite, and it is assumed that individuals of a given species share a common strategy (i.e. they behave either as rock, paper, or scissor and do not change). So the game is played among species, but over numerous of individuals interacting with each other, and the unpredictability lies in the random encounters of individuals of different species at one place and time. Does intransitivity in competitive relationships among species indeed result in stable coexistence?

With life spans of hundreds of years, this question is hard to investigate empirically for tropical trees. Bacteria, however, with well-defined characteristics and a short generation time, have shown to be interesting model systems for testing questions of species coexistence (Hol and Dekker 2014; Kerr et al. 2002). And the answer is no, intransitivity all by itself does not necessarily lead to coexistence. Kerr et al. (2002) carried out a compelling test with the model bacteria Escherichia coli. Three strains that together constitute an intransitive competitive network were grown in mixtures. One strain produces the toxin colicin (colicinogenic cells C), to which other strains are either sensitive (sensitive cells $\mathrm{S}$ ) or immune (resistant cells R). Colicin production, and to a lesser degree immunity, is costly to the cells and compromises the growth rates of the $\mathrm{C}$ and $\mathrm{R}$ strains. As a result, this $\mathrm{C}-\mathrm{S}$ - $\mathrm{R}$ system satisfies the rock-paper-scissor relationship because $\mathrm{C}$ can displace $\mathrm{S}$ (because $\mathrm{C}$ kills $\mathrm{S}$ ), $\mathrm{R}$ can displace $\mathrm{C}$ (because $\mathrm{R}$ has a growth-rate advantage) and $\mathrm{S}$ can displace $\mathrm{R}$ (because $\mathrm{S}$ has a growth-rate advantage) (Kerr et al. 2002). When all three strains were mixed together in liquid medium in a flask and shaken, maximizing interactions among the bacteria, the $\mathrm{S}$ strain was rapidly driven to extinction by $\mathrm{C}$, and subsequently $\mathrm{C}$ was outcompeted by $\mathrm{R}$ due to the higher growth rate of the latter.

Why do the strains fail to show coexistence, although the conditions of intransitivity are met? Theoretical models predict that if the competitive relationships are transitive, but the dynamics lead to different strengths of interaction, fluctuations may appear and one strategy may eventually win (Nowak and Sigmund 2004). In the case of $E$. coli, the toxin produced by the C strain is immediately lethal to the $\mathrm{S}$ strain, but the growth advantage of the $\mathrm{S}$ strain over the other two strains results in slower replacement. Consequently, when the communities interact 'globally' in a shaken flask, $\mathrm{S}$ is eradicated quickly and the intransitive network collapses.

Interestingly, Kerr et al. (2002) showed that when interactions among the three types of bacteria were not global but local, at the surface of a petri dish filled with 
agar, coexistence did occur. Here encounters were no longer random because the strains occurred in patches (clumps) and interacted at the borders where patches of different strains met. Pictures of the petri dishes over time show that strains were chasing each other, as predicted by the rock-paper-scissor relationships, resulting in a pattern of clumps that is changing all the time. Kerr et al. (2002) concluded that "balanced chasing in a spatially structured, non-hierarchical community may result in the maintenance of diversity". Spatial structure where individuals with similar strategy clump and limited dispersal may give much better chances for the maintenance of diversity than well-mixed populations (Nowak and Sigmund 2004).

\subsection{Global Stability in Hyper-Diverse Plant Communities Consistent with Local Rock-Paper-Scissor Dynamics}

To what extent is this coexistence mechanism also to be expected for hyper-diverse communities of tropical forest or grassland? An increasing number of studies have shown that competitive relationships between plant species are not hierarchical but intransitive (de Kroon et al. 2012; Soliveres et al. 2015). Dynamics are obviously orders of magnitude slower than in bacterial communities but, interestingly, long-term observations have revealed patchy dynamics of grassland species that are reminiscent of those of the bacterial patches in petri dishes. In grasslands at the slopes of the Krkonoše mountains in the Czech Republic, species form patches that change position all the time because individuals die and are replaced by other species at one location while they appear at locations nearby as a result of clonal expansion or germination (Herben et al. 1993a). The replacements of species are largely random and to some degree intransitive and thus resemble the "balanced chasing" described above (Herben et al. 1997; Herben et al. 1993b). The consequence is a very stable community as a whole, while paradoxically numerous replacements take place at a local scale. Indeed, a $10 \times 10 \mathrm{~m}$ area of these grasslands would look very much the same with the same species co-occurring year after year, but if one could make a movie of the area over decades the species would be seen to move around like ants in an ants nest. For tropical forest, the Janzen-Connell hypothesis predicts very similar spatial dynamics. Although there is a huge number of trees in a tropical forest, a particular tree will interact most with its direct neighbours, while dispersal distances are limited in most cases. However, replacements are even slower than in grasslands. The oldest forest dynamics plot at Barro Colorado Island in Panama (where all trees of all species over 50 ha are mapped; Condit 1995) was laid out in the early 1980s and is still way too young for a demonstration of such dynamics. 


\subsection{Global Stability Through Neutral Dynamics if Species Are Demographically Equal}

The growth rate differences between the bacterial strains of E. coli in the study of Kerr et al. (2002) hinge upon an important element in current coexistence theory, i.e. fitness differences between species (Chesson 2000). As explained above, the key stabilizing force in communities are the niche differences, the fundamental requirements between species affecting their population growth rates. Because of these differences, individuals of the same species have stronger competitive interactions than individuals of different species. In other words, species limit their own growth more than they limit the growth of other species, i.e. intraspecific competition is stronger than interspecific competition. The degree to which intra- and interspecific competition coefficients must be different for stable coexistence to occur depends on the average fitness differences between species (Adler et al. 2007; Chesson and Kuang 2008). Fitness in this context refers to the relative degree of adaptedness of that species to the conditions of the habitat in the absence of niche differences (Chesson 2011). Species with higher fitness develop higher population growth rates and will win the competition. Niche differences balance the fitness differences stabilizing the dynamics and providing the conditions for coexistence.

Coexistence will also be promoted not only if niche differences are larger, but also if fitness differences are smaller. Spatial structure is one way to reduce the effects of fitness differences, as the examples of $E$. coli and grasslands illustrate. Indeed, competitive replacement may be slowed down considerably if competitors are growing in patches, with interspecific interactions taking place at the border. In such cases, most of the interactions in the community are between members of the same species, rather than between members of different species, favoring the weaker competitor (Stoll and Prati 2001). Spatial structure and limited dispersal, both prominent in most ecosystems, are thus forces that equalize fitness differences between species and promote coexistence.

The most radical and influential idea with respect to the maintenance of species diversity has been the formulation of 'neutral theory' (Hubbell 2001). Neutral theory essentially states that all species are demographically equal, i.e. that fitness differences do not exist. Species may differ in numerous traits and resource requirements, but do not translate into a net difference in population growth rates between the species under prevailing habitat conditions. In this theory, there are no niche differences and there is no stable coexistence, but there is an opportunity for long-time co-occurrence. Individuals do compete for limited resources but competitive strengths are similar for all individuals, irrespective of species identity, resulting in replacements driven by chance. All these random replacements add up to neutral dynamics in which populations of different species are maintained if the community is of sufficient size. Population numbers do fluctuate as a result of stochastic (e.g. climatic) influences and are not buffered against extinction. Particularly in communities of limited size, such as in fragmented habitats, 
populations have a chance of going extinct under neutral dynamics due to demographic stochasticity (as further explained in de second example below).

When published in 2001, Hubbell's book was a provocation to the many community ecologists studying niche axes in their communities. Fifteen years later, neutral theory has been shown to predict community characteristics surprisingly well (Rosindell et al. 2011). It has been accepted as an inherent element in community theory and not only in the tropical forest that formed its inspiration. Indeed, also in species-rich grasslands much of the competitive interactions appear largely equivalent among species (Law et al. 1997), resulting in random replacements at the local scale, and near neutral dynamics at the larger scale of the community, despite the fact that we know that these species differ in their ecological requirements.

\subsection{Coexistence Mechanisms May Result in Unpredictable Dynamics}

While consensus is now emerging about how neutral and niche processes together govern community dynamics, we should realize that they do not necessarily result in overall community stability. Ground-breaking mathematical theory developed in the 1970's by Robert May, showed that simple differential equations with density-dependent feedback could result in very complex non-linear dynamics of the system with chaotic fluctuations that are by definition unpredictable (May 1976; Weitzel and Rosenkranz, this volume). Work of Jef Huisman and co-workers has demonstrated that such dynamics bear relevance for the coexistence of many species of plankton in aquatic ecosystems. Huisman and Weissing (1999) showed theoretically that a well-parameterised competition model, describing the competition for limiting resources (such as nitrogen, phosphorus, silicon, light and inorganic carbon) gave rise to coexistence of many different species of plankton. The number of species coexisting was much more than expected on the basis of their differences in resource requirements as predicted by the competitive exclusion principle (i.e. their niche differences alone). The model predicted that the species displaced each other in a cyclic fashion, giving rise to oscillations and chaotic dynamics, reminiscent of the non-linear dynamics described by May (1976). Despite sometimes major fluctuations in species numbers, when a species became dominant, other species at low numbers bounced back, though at different rates. Later empirical work eloquently demonstrated that these predictions may actually occur in reality (Benincà et al. 2008). In a laboratory setup with a plankton community in tanks many different species coexisted for a period up to 2300 days, covering a couple of hundred generations. They did so while showing population size fluctuations over several orders of magnitude that were essentially unpredictable, yet leading to overall persistence of the community. Note that the conditions were stable and there was no spatial structure within the tanks, all plankton species interacted with random encounters (as in the flasks of Kerr et al. 2002). The chaotic fluctuations were 
attributed to different species interactions in the planktonic food web, giving rise to different periodicities in the ups and downs of the various populations. It is important to realize that these dynamics arise from inherent deterministic relationships, i.e. from competition and predation process (Huisman and Weissing 1999).

Recently, Benincà et al. (2015) demonstrated for the first time that near-chaotic dynamics may occur in the world outside. In an intertidal ecosystem in New Zealand, cyclic replacements occur of barnacles colonizing bare rock, brown alga overgrowing barnacles, mussels settling on barnacles and algae, giving rise to bare rock as the mussels eventually detach. The cyclic fluctuations of the populations in this community become irregular through the seasonality of the system and the time needed for the establishment of each of the species. Interestingly, the cyclic replacement is reminiscent of rock-paper-scissor interactions (Benincà et al. 2015) but the dynamics do not resemble those of the E. coli strains described above (Kerr et al. 2002). The reason might be that with sessile stages but global dispersal of recruits the intertidal community is neither global (leading to the dominance of a single species in the well-mixed flasks), nor completely spatially structured (resulting in balanced chasing at patch edges and overall stability).

\subsection{Conclusion: The Interplay Between Scale-Dependent Predictable and Unpredictable Patterns in Community Dynamics}

We have seen three archetypes of long-term persistence of complex communities with many different species, all operating in a very different way. In the case of niche differences between species (as in the case of rock-paper-scissor games), stabilizing forces may be strong and promote stability. Such communities are likely spatio-temporally structured with many predictable species replacements at a local scale. Neutral theory confronts us with the situation that numerous random interactions at a local scale sum up to stochastic dynamics at a global scale. However, in a relatively stable environment, competitive equivalence among species, as assumed in neutral theory, can lead to overall predictable community patterns (long-term co-occurrence). Finally, the plankton example shows the reverse, where predictable species replacements result in chaotic dynamics of a community which is as unpredictable as the weather (Benincà et al. 2008).

These contrasts feed the uneasy relationship that ecology has with deterministic versus stochastic processes underlying the structure of ecological communities (Bjørnstad 2015; Chase and Myers 2011; Vellend et al. 2014). Is any fundamental process really stochastic, or does it always have an underlying deterministic origin? These examples indicate that the scale of processes must be considered, with many different possibilities. Truly stochastic (i.e. unpredictable) dynamics may find their origin in underlying deterministic processes, while stochastic interactions at a local 
scale may give rise to relatively stable (and hence predictable) dynamics at a global scale. Each scale calls for its own methodologies describing processes and dynamics (Vellend et al. 2014). It is important to understand the interplay of deterministic vs. stochastic processes with the scales of organisation, as it affects the nature of causality in ecology. We will see this now in our second example.

\section{Example 2: Understanding Species Population Trends}

\subsection{Species Survive in Metapopulations with a High Incidence of Chance Effects}

In the current fragmented landscape, in almost all regions of the world, species are distributed in discrete populations. At some point in time every single population started with a colonization event of an area where the species did not occur at that moment, and after a while (which may take days, years, or centuries) every population will go extinct when the last individual has died or left the area. The Finnish ecologist Illka Hanski coined the collection of discrete populations in the landscape a metapopulation (Hanski 1998). The success of a species is defined by its metapopulation dynamics, determined by the processes of immigration and extinction. The classical example, and the one where metapopulations were first described, is the Baltic Sea with numerous islands for the coast of Finland inhabited by butterflies that form small populations on the islands, connected by dispersal of the butterflies between the islands. Hanski et al. (1994) demonstrated how the islands are colonized and vacated by the butterflies, leading to continuous changes in island occupation, but with remarkable stability of the metapopulation of butterflies in the archipelago.

The success or decline of species is described by the fluctuations in the metapopulation as a whole. These global fluctuations are the accumulation of numerous extinction and colonisation events at the local scale. As favourable habitat may be small (as is the case for many of the islands in the Finnish archipelago) chance effects play a large role. Any local population can be subject to accidental hazards such as a fire or storm leading to local extinction. The local unpredictable variation is referred to as environmental stochasticity (Lande 1993). In addition, demographic stochasticity exists whereby small populations can simply go extinct due to chance effects (Lande 1993). The smaller the population, the bigger the chance that all individuals leave the local habitat, die, or fail to reproduce, partly due to difficulties of finding mates and/or inbreeding depression. Colonisation events, whereby unoccupied habitat is discovered by animals from elsewhere, also have a high element of chance (unless dispersers actively search for empty habitat). 


\subsection{Farmland Birds: Understanding Population Trends}

An urgent question nowadays is to what extent climate change, pollution, or changes in land use form a threat to species of plants and animals (Bowler et al. 2015). And if so, can negative effects be mitigated? But how can we understand these threats if species survive in metapopulations, where the dynamics are the cumulative effects of numerous events where chance plays a major role? How can we control this unpredictable variation, identify and quantify causes of decline, and suggest measures to counteract the threats? As in our previous example, we deal with local processes scaling up to patterns at larger scales, and stochastic local dynamics leading to global stability.

We illustrate these questions with an example of the status of farmland birds in the Netherlands and its association with neonicotinoid insecticides in the environment (Hallmann et al. 2014). Farmland bird species in the Western landscape have been decimated over the last hundred years as a result of agricultural intensification, increased fertilisation and pesticide use. Many bird populations are now confined to small suitable habitat patches like hedgerows, or along water bodies, often consisting of only few bird territories. Bird territories in the Netherlands are counted in a standardized way by thousands of volunteers under auspices of Sovon, the Dutch Centre for Field Ornithology. Bringing this information together we know that, over recent decades, some bird species in the Netherlands show signs of recovery, albeit not to the same extent throughout the country. Zooming in, we typically see a patchwork across the Netherlands with local areas in which bird populations increase, interspersed with areas with negative population trends. Investigating 15 insectivorous farmland bird species, Hallmann et al. (2014) demonstrated that these differences correlated with the local concentration of imidacloprid in the surface water. Imidacloprid is the most widely use neonicotinoid, a group of insecticides introduced in the mid-nineties. Neonicotinoids specifically target the nervous system of insects and are therefore highly lethal to invertebrates and much less so to vertebrates like humans or birds. Applied as seed coating or by spraying, major quantities of insecticides are not taken up by the crop to be protected but wash out in the soils and accumulate in the waterways. It is in these environments that larval stages of insects grow up, which form the bulk food of the bird species investigated. Hallmann et al. therefore hypothesised that local populations of these bird species, relying on insects particularly in the breeding season, are in decline due to food shortage.

Hallmann et al.'s study is essentially correlative, showing that local bird population trends are more likely to be negative when local imidacloprid concentrations in the surface water are higher. The case for imidacloprid as a cause of bird decline was reinforced in two ways. First, local bird trends over the last ten years were also correlated to local changes in land use that were known to affect bird populations, including changes in levels of nitrogen use, and changes in areas of maize, winter cereals, fallow land, greenhouses and alike. In this analysis imidacloprid stood out as by far the best explanatory variable for local bird population trends. Second, the 
correlation between imidacloprid and bird trends was much weaker and non-significant when bird trends in the same areas were considered before the introduction of the compound, suggesting that the correlation was not due to some unknown explanatory variable already present before the introduction of neonicotinoids.

\subsection{Mastering Chance Effects at Local Scale to Explain Global Trends}

Nevertheless, the Hallmann et al. (2014) study remains correlative, raising the pertinent question: Are neonicotinoids the causal factor for the trends in bird decline? In the worldwide press attention that the study received, this question came up repeatedly. In a response to Hallman et al., the Dutch Minister of the Environment expressed her concern about the effects of neonicotinoids on the environment, but also said that changes in legislation could not be based on a correlative study only. In the strict sense, demonstrating causality would require experiments (Dively et al. 2015; Godfray et al. 2014; Rundlof et al. 2015) but for birds this would entail field trials at such a large spatial scale and over such a long time that they are impossible to conduct. We therefore must find other ways to demonstrate causality. This is very difficult because, as explained above, mechanisms of colonisation, growth and extinction of the populations operate at a small spatial scale, where chance processes and other local factors may prevail. Indeed, even the interpretation of correlations at the global (metapopulation) scale in the context of local effects can lead to apparent contradictions of the kind we also see in epidemiology.

Emerging global trends such as the bird trends in relation to imidacloprid may not necessarily be seen locally everywhere. At the scale of the Netherlands, the trend was quite strong: bird populations declined with a rate of $3.5 \%$ per year where local imidacloprid concentrations exceeded $20 \mathrm{ng} / \mathrm{l}$ in the surface water. While this trend was highly significant, part of the variation in bird trends remained unexplained, and appeared as noise around the correlation. Some of this variation may be due to chance effects related to environmental and stochastic stochasticity. But, how then can a population escape the hazards of neonicotinoids at a local scale? This remains to be investigated, but as an example the following situation can easily be envisioned. Imagine an agricultural field adjacent to the dunes, which are important nature reserves for birds in the Netherlands. If the insecticide pollutes the local soil and waterways and deteriorates local insect populations, birds in the vicinity may be little affected as they can forage in the dune area with its own hydrology, not affected by the pollution nearby. Such a population may be healthy and increasing, while the overall analysis predicts a declining population at this location with a high imidacloprid load. However, given enough data at the scale of 
the Netherlands, the 'noise' of such local situations will not mask a general correlation between bird trends and insecticide concentrations.

Throughout ecology such unexplained variation is rather common: ecosystems are influenced by a very large number of factors at the same time. Ecologists therefore strive to quantify more and more of the environmental factors that influence e.g. bird trends, or at least try to determine what the most important explanatory factors are. But even when we understand a fair amount of what drives local populations (50\% of variation explained is certainly a glass well filled for ecologists), the mechanisms (and chance events) of dispersal between areas remain even more elusive. Recent attempts to model dispersal focus more and more on all aspects of dispersal: what local conditions lead to the initiation of dispersal, how far does an individual travel through a landscape, what makes him/her stop, and what is the impact at the destination? Combining spatial population models and mechanistic 'gravity' models of dispersal (reviewed in Jongejans et al. 2015) might therefore be a way forward in linking local processes and global trends, although unexplained variation will remain (due to chance but also due to unmeasured factors).

\subsection{Understanding Causality: A Comparison with Epidemiology}

Ecology is not the only field of research that struggles with the reconciliation of processes and patterns that are apparent (or not) at different scales. Similar difficulties in relating global trends to local effects appear in epidemiology. Epidemiological studies investigate large groups of people and identify the factors that may explain the differences in health. Well-known examples include how smoking is related to human mortality (Banks et al. 2015; Thun et al. 2013), and how obesity (and diet) is related to an increased chance of diseases and premature death (references in Würtz et al. 2014). However, as we all know, 'local' exceptions to these convincing 'global' trends exist. Many people are acquainted with a person reaching old age in relatively good health while smoking like a chimney. We can ask a similar question as with the bird example: how can a person escape the hazard of smoking? Detailed investigation of the medical condition and the habits of such a person could perhaps give indications. If unsuccessful we consider the health of the person as a happy coincidence, but this would not dismiss the hazards of smoking in general. Still, the global trends between smoking and mortality remain essentially a correlation.

As with our bird study, proper controlled experiments on humans regarding effects of unhealthy diet or smoking are considered unethical and cannot be done. Epidemiology has long recognized the weakness of the correlative nature of its investigations for identifying the biological and behavioural causes of disease (Galea et al. 2010; Hill 1965; March and Susser 2006). Still, it is possible to discover cause-effect relationships from purely observational data (Pearl and Verma 
1991). Current methodologies attempt to solve this problem by capturing the complexity of the many risk factors for human health in complex systems modelling (Galea et al. 2010). Another way forward is advanced statistics on large datasets together with targeted measurements. Human metabolic profiles are important health indicators and an important question is to what extent they are related to Body Mass Index (BMI) or to a genetic disposition for adiposity, even for people in the non-obese range. By using a Mendelian randomisation framework, Würtz et al. (2014) have recently shown how health indicators can be causally related to BMI, by incorporating a gene score for predisposition to elevated BMI. This statistical method is designed to infer causality in observational studies while taking possible confounding effects into account.

Similar to the situation in epidemiology, observational studies in ecology are often the only field instruments to gauge the 'health' of populations of species of conservation interest. Statistical and modelling techniques are only beginning to be applied to master the explained against the unexplained variation, and to quantify causation taking into account the many confounding factors operating at different scales. Further work in this direction is required to convince public and decision makers that effects are real and require appropriate action. The history of the implementation of smoke restrictions indicates that this is not an easy trajectory.

\section{Epilogue}

Chance is pervasive in ecological systems. However, chance events never come alone. They may have a solid deterministic origin or they may scale up to predictable variation. In many cases stochasticity and determinism are closely intertwined through the different scales of biological organisation. The scale-dependency of cause and effect has an uneasy relationship with the scale-dependency of stochasticity and determinism. We should take this relationship into account when defining causality, as well as in what can be considered as scientific proof. Methods are to be developed to quantify causal relationships and distinguish them from random effects and confounding factors.

Acknowledgements This essay was inspired by the excellent work of our graduate students Caspar Hallmann, Marloes Hendriks, Janneke Ravenek and Marco Visser. We are grateful to our collaborators Ruud Foppen, Tomas Herben, Liesje Mommer, Helene Muller-Landau, Chris van Turnhout, Wim van der Putten and Joe Wright for enlightening discussion over the years.

Open Access This chapter is distributed under the terms of the Creative Commons Attribution-Noncommercial 2.5 License (http://creativecommons.org/licenses/by-nc/2.5/) which permits any noncommercial use, distribution, and reproduction in any medium, provided the original author(s) and source are credited. The images or other third party material in this chapter are included in the work's Creative Commons license, unless indicated otherwise in the credit line; if such material is not included in the work's Creative Commons license and the respective action is not permitted by statutory regulation, users will need to obtain permission from the license holder to duplicate, adapt or reproduce the material. 


\section{References}

Adler, P. B., HilleRisLambers, J., \& Levine, J. M. (2007). A niche for neutrality. Ecology Letters, 10, 95-104.

Banks, E., Joshy, G., Weber, M. F., Liu, B., Grenfell, R., Egger, S., et al. (2015). Tobacco smoking and all-cause mortality in a large Australian cohort study: Findings from a mature epidemic with current low smoking prevalence. BMC Medicine, 13, 281.

Benincà, E., Ballantine, B., Ellner, S. P., \& Huisman, J. (2015). Species fluctuations sustained by a cyclic succession at the edge of chaos. Proceedings of the National Academy of Sciences of the United States of America, 112, 6389-6394.

Benincà, E., Huisman, J., Heerkloss, R., Jöhnk, K. D., Branco, P., Van Nes, E. H., et al. (2008). Chaos in a long-term experiment with a plankton community. Nature, 451, 822-825.

Bever, J. D., Platt, T. G., \& Morton, E. R. (2012). Microbial population and community dynamics on plant roots and their feedbacks on plant communities. Annual Review of Microbiology, 66, 265-283.

Bjørnstad, O. N. (2015). Nonlinearity and chaos in ecological dynamics revisited. Proceedings of the National Academy of Sciences of the United States of America, 112, 6252-6253.

Bowler, D., Haase, P., Kröncke, I., Tackenberg, O., Bauer, H., Brendel, C., et al. (2015). A cross-taxon analysis of the impact of climate change on abundance trends in central Europe. Biological Conservation, 187, 41-50.

Buss, L. W. (1980). Competitive intransitivity and size-frequency distributions of interacting populations. Proceedings of the National Academy of Sciences of the United States of America-Biological Sciences, 77, 5355-5359.

Chase, J. M., \& Myers, J. A. (2011). Disentangling the importance of ecological niches from stochastic processes across scales. Philosophical Transactions of the Royal Society B: Biological Sciences, 366, 2351-2363.

Chesson, P. (2000). Mechanisms of maintenance of species diversity. Annual Review of Ecology and Systematics, 31, 343-366.

Chesson, P. (2011). Ecological niches and diversity maintenance. In I. Pavlinov (Ed.), Research in Biodiversity —Models and Applications. Rijeka: In Tech.

Chesson, P., \& Kuang, J. J. (2008). The interaction between predation and competition. Nature, $456,235-238$.

Comita, L. S., Queenborough, S. A., Murphy, S. J., Eck, J. L., Xu, K., Krishnadas, M., et al. (2014). Testing predictions of the Janzen-Connell hypothesis: A meta-analysis of experimental evidence for distance- and density-dependent seed and seedling survival. Journal of Ecology, $102,845-856$.

Condit, R. (1995). Research in large, long-term tropical forest plots. Trends in Ecology \& Evolution, 10, 18-22.

Connell, J. H. (1971). On the role of natural enemies in preventing competitive exclusion in some marine animals and in rain forest trees. In P. J. den Boer \& G. R. Gradwell (Eds.), Dynamics of populations (pp. 298-312). Wageningen: Center for Agricultural Publishing and Documentation.

de Kroon, H., Hendriks, M., van Ruijven, J., Ravenek, J., Padilla, F. M., Jongejans, E., et al. (2012). Root responses to nutrients and soil biota: Drivers of species coexistence and ecosystem productivity. Journal of Ecology, 100, 6-15.

Dively, G. P., Embrey, M. S., Kamel, A., Hawthorne, D. J., \& Pettis, J. S. (2015). Assessment of chronic sublethal effects of imidacloprid on honey bee colony health. PLoS One, 10, e0118748.

Galea, S., Riddle, M., \& Kaplan, G. A. (2010). Causal thinking and complex system approaches in epidemiology. International Journal of Epidemiology, 39, 97-106.

Gilpin, M. E. (1975). Limit cycles in competition communities. American Naturalist, 109, 51-60.

Godfray, H. C. J., Blacquiere, T., Field, L. M., Hails, R. S., Petrokofsky, G., Potts, S. G., et al. (2014). A restatement of the natural science evidence base concerning neonicotinoid 
insecticides and insect pollinators. Proceedings of the Royal Society of London B: Biological Sciences, 281(1786), 20140558.

Hallmann, C. A., Foppen, R. P. B., van Turnhout, C. A. M., de Kroon, H., \& Jongejans, E. (2014). Declines in insectivorous birds are associated with high neonicotinoid concentrations. Nature, $511,341-343$.

Hanski, I. (1998). Metapopulation dynamics. Nature, 396, 41-49.

Hanski, I., Kuussaari, M., \& Nieminen, M. (1994). Metapopulation structure and migration in the butterfly Melitaea cinxia. Ecology, 75, 747-762.

Herben, T., Krahulec, F., Hadincova, V., \& Kovarova, M. (1993a). Small-scale spatial dynamics of plant species in a grassland community over six years. Journal of Vegetation Science, 4, $171-178$.

Herben, T., Krahulec, F., Hadincová, V., Pechacková, S., \& Kovárová, M. (1997). Fine-scale spatio-temporal patterns in a mountain grassland: Do species replace each other in a regular fashion? Journal of Vegetation Science, 8, 217-224.

Herben, T., Krahulec, F., Hadincová, V., \& Skálová, H. (1993b). Small-scale variability as a mechanism for large-scale stability in mountain grasslands. Journal of Vegetation Science, 4, $163-170$.

Hill, A. B. (1965). Environment and disease-association or causation? Proceedings of the Royal Society of Medicine-London, 58, 295-300.

Hol, F. J., \& Dekker, C. (2014). Zooming into see the bigger picture: Microfluidic and nanofabrication tools to study bacteria. Science, 346, 1251821.

Hubbell, S. P. (2001). The unified neutral theory of biodiversity and biogeography. Princeton: Princeton University Press.

Huisman, J., \& Weissing, F. J. (1999). Biodiversity of plankton by species oscillations and chaos. Nature, 402, 407-410.

Isard, S., Barnes, C., Hambleton, S., Ariatti, A., Russo, J., Tenuta, A., et al. (2011). Predicting soybean rust incursions into the North American continental interior using crop monitoring, spore trapping, and aerobiological modeling. Plant Disease, 95, 1346-1357.

Janzen, D. H. (1970). Herbivores and the number of tree species in tropical forests. American Naturalist, 104, 501-528.

Jongejans, E., Skarpaas, O., Ferrari, M. J., Long, E. S., Dauer, J. T., Schwarz, C. M., et al. (2015). A unifying gravity framework for dispersal. Theoretical Ecology, 8, 207-223.

Kerr, B., Riley, M. A., Feldman, M. W., \& Bohannan, B. J. M. (2002). Local dispersal promotes biodiversity in a real-life game of rock-paper-scissors. Nature, 418, 171-174.

Laird, R. A., \& Schamp, B. S. (2006). Competitive intransitivity promotes species coexistence. American Naturalist, 168, 182-193.

Lande, R. (1993). Risks of population extinction from demographic and environmental stochasticity and random catastrophes. American Naturalist, 142, 911-927.

Lankau, R. A. (2010). Intraspecific variation in allelochemistry determines an invasive species' impact on soil microbial communities. Oecologia, 165, 453-463.

Lankau, R. A., Wheeler, E., Bennett, A. E., \& Strauss, S. Y. (2011). Plant-soil feedbacks contribute to an intransitive competitive network that promotes both genetic and species diversity. Journal of Ecology, 99, 176-185.

Latham, R. E., \& Ricklefs, R. E. (1993). Global patterns of tree species richness in moist forestsEnergy-diversity theory does not account for variation in species richness. Oikos, 67, 325-333.

Law, R., Herben, T., \& Dieckmann, U. (1997). Non manipulative estimates of competition coefficients in a montane grassland community. Journal of Ecology, 85, 505-517.

Lotka, A. J. (1920). Analytical note on certain rhythmic relations in organic systems. Proceedings of the National Academy of Sciences of the United States of America, 6, 410-415.

Mangan, S. A., Schnitzer, S. A., Herre, E. A., Mack, K. M. L., Valencia, M. C., Sanchez, E. I., et al. (2010). Negative plant-soil feedback predicts tree-species relative abundance in a tropical forest. Nature, 466, 752-755.

March, D., \& Susser, E. (2006). The eco- in eco-epidemiology. International Journal of Epidemiology, 35, 1379-1383. 
May, R. M. (1976). Simple mathematical models with very complicated dynamics. Nature, 261, 459-467.

McGill, B. J. (2010). Matters of scale. Science, 328, 575-576.

Nowak, M. A., \& Sigmund, K. (2004). Evolutionary dynamics of biological games. Science, 303, 793-799.

Pearl, J., \& Verma, T. (1991). A theory of inferred causation. In J. Allen, R. Fikes \& E. Sandewall (Eds.), Knowledge Representation and Reasoning: Proceedings of the Second International Conference. San Mateo, CA: Morgan Kaufmann.

Petermann, J. S., Fergus, A. J. F., Turnbull, L. A., \& Schmid, B. (2008). Janzen-Connell effects are widespread and strong enough to maintain diversity in grasslands. Ecology, 89, 2399-2406.

Rosindell, J., Hubbell, S. P., \& Etienne, R. S. (2011). The unified neutral theory of biodiversity and biogeography at age ten. Trends in Ecology \& Evolution, 26, 340-348.

Rundlof, M., Andersson, G. K., Bommarco, R., Fries, I., Hederstrom, V., Herbertsson, L., et al. (2015). Seed coating with a neonicotinoid insecticide negatively affects wild bees. Nature, 521, 77-80.

Slik, J. F., Arroyo-Rodríguez, V., Aiba, S.-I., Alvarez-Loayza, P., Alves, L. F., Ashton, P., et al. (2015). An estimate of the number of tropical tree species. Proceedings of the National Academy of Sciences of the United States of America, 201423147.

Soliveres, S., Maestre, F. T., Ulrich, W., Manning, P., Boch, S., Bowker, M. A., et al. (2015). Intransitive competition is widespread in plant communities and maintains their species richness. Ecology Letters, 18(8), 790-808.

Stoll, P., \& Prati, D. (2001). Intraspecific aggregation alters competitive interactions in experimental plant communities. Ecology, 82, 319-327.

Thun, M. J., Carter, B. D., Feskanich, D., Freedman, N. D., Prentice, R., Lopez, A. D., et al. (2013). 50-year trends in smoking-related mortality in the United States. New England Journal of Medicine, 368, 351-364.

Vellend, M., Srivastava, D. S., Anderson, K. M., Brown, C. D., Jankowski, J. E., Kleynhans, E. J., et al. (2014). Assessing the relative importance of neutral stochasticity in ecological communities. Oikos, 123, 1420-1430.

Volterra, V. (1928). Variations and fluctuations of the number of individuals in animal species living together. Journal du Conseil. Conseil Permanent International pour l'Exploration de la Mer, 3, 3-51.

Wilson, J. B., Peet, R. K., Dengler, J., \& Pärtel, M. (2012). Plant species richness: The world records. Journal of Vegetation Science, 23, 796-802.

Würtz, P., Wang, Q., Kangas, A. J., Richmond, R. C., Skarp, J., Tiainen, M., et al. (2014). Metabolic signatures of adiposity in young adults: Mendelian randomization analysis and effects of weight change. PLoS Medicine, 11, e1001765. 